\title{
Resistencia emergente a la mupirocina en aislados de Staphylococcus aureus resistente a meticilina en un hospital pediátrico terciario en la Argentina
} Emerging mupirocin resistance in methicillin-resistant Staphylococcus aureus
isolates at a tertiary care children's hospital in Argentina

\author{
Lic. Nicolás M. Vázqueza , Farm. Paulo Cáceres Guidob, Mg. Graciela Fiorillic y Dra. Silvia Moreno ${ }^{a}$
}

\begin{abstract}
RESUMEN
En América Latina, existen escasos estudios sobre la resistencia a mupirocina y producción de biofilm en Staphylococcus aureus resistente a la meticilina (SARM). En este trabajo, se investigó la resistencia a mupirocina en SARM aislados de pacientes pediátricos con bacteremia y su capacidad para producir biofilm. Se estudió la resistencia a antibióticos por Kirby-Bauer y microdilución en caldo. Se cuantificó el biofilm bacteriano por ensayo de cristal violeta. El 2,3\% (5/217) de los aislados deSARM presentaron un alto nivel de resistencia a mupirocina con una concentración inhibitoria mínima de $>512 \mu \mathrm{g} / \mathrm{ml}$, además de resistencia cruzada con clindamicina, eritromicina, gentamicina y ciprofloxacina. Notablemente, dichos aislados formaron biofilm en un nivel moderado-alto. Este primer reporte en Argentina de aislados clínicos de SARM resistentes a la mupirocina es clave para seguir su evolución en el tiempo a nivel local y en la región de América Latina.

Palabras clave: Staphylococcus aureus, resistencia a medicamentos, mupirocina, biofilm, pediatría.
\end{abstract}

http: / / dx.doi.org/10.5546/ aap.2019.48

Texto completo en inglés:

http: / / dx.doi.org/10.5546/ aap.2019.eng.48

Cómo citar: Vázquez NM, Cáceres Guido P, Fiorilli G, Moreno S. Resistencia emergente a la mupirocina en aislados de Staphylococcus aureus resistente a meticilina en un hospital pediátrico terciario en la Argentina. Arch Argent Pediatr 2019;117(1):48-52.

a. Laboratorio de Farmacología de Activos Vegetales, Centro de Estudios Biomédicos, Biotecnológicos, Ambientales y de Diagnóstico (CEBBAD), CONICET, carreras de Farmacia y Bioquímica. Universidad Maimónides.

b. Grupo de Medicina Integradora. Hospital de Pediatría Garrahan, Ciudad Autónoma de Buenos Aires, Argentina.

c. Servicio de Microbiología.

Hospital de Pediatría Garrahan.

Ciudad Autónoma de Buenos Aires, Argentina.

Correspondencia:

Dra. Silvia Moreno: smorenocontar@gmail.com

Financiamiento: Esta investigación fue financiada por el Consejo Nacional de Investigaciones Científicas y Técnicas (CONICET). Beca: Proyectos de Investigación Plurianuales (PIP) 00894 (Dra. Silvia Moreno) y Universidad Maimónides.

Conflicto de intereses: Ninguno que declarar.

Recibido: 23-3-2018

Aceptado: 30-7-2018

\section{GLOSARIO}

CIM: Concentración inhibitoria mínima.

CV: Cristal violeta.

DO: Densidad óptica.

DOc: DO crítica.

SARM: Staphylococcus aureus resistente a la meticilina. SARM-R-MUP: SARM resistente a mupirocina. SARM-S-MUP: SARM susceptible a mupirocina .

\section{INTRODUCCIÓN}

Las infecciones estafilocócicas comunes en los tejidos blandos se asocian también con altas tasas de morbilidad y mortalidad en muchos países del mundo, incluida Argentina. ${ }^{1,2}$ En particular, las infecciones asociadas con $S$. aureus resistente a la meticilina (SARM) ponen en peligro la vida, especialmente, en los huéspedes inmunosuprimidos, cuando la bacteria se propaga desde la piel a los pulmones, al torrente sanguíneo o a otros órganos del cuerpo. ${ }^{2}$ Por esa razón, en los pacientes hospitalizados la descolonización con un agente antimicrobiano tópico, como la mupirocina, se usa comúnmente para evitar la diseminación del SARM. ${ }^{3}$ Sin embargo, la efectividad de dicho tratamiento se vio disminuida de manera drástica por la aparición de aislados resistentes. ${ }^{4}$ Se han encontrado aislados con bajo o alto nivel de resistencia a la mupirocina, definida según la concentración inhibitoria mínima (CIM) observada (8-256 $\mu \mathrm{g} /$ $\mathrm{ml} \mathrm{o}>512 \mu \mathrm{g} / \mathrm{ml}$, respectivamente). ${ }^{5}$ Otro factor que contribuye al éxito del $S$. aureus como patógeno humano en los entornos de atención médica es su capacidad para la formación del biofilm bacteriano, comunidades multicelulares de células adheridas a una superficie, como los dispositivos médicos o los tejidos. Las infecciones recurrentes y crónicas por SARM asociadas con el desarrollo del biofilm tienen un gran impacto en la selección de regímenes terapéuticos. ${ }^{6}$

Existen pocos estudios en Argentina y en América Latina sobre la caracterización del SARM 
en los pacientes pediátricos. ${ }^{7,8}$ Asimismo, mucho menos se sabe sobre la presencia de la resistencia a la mupirocina y la capacidad de formación de biofilm en SARM. El objetivo fue determinar la presencia de SARM resistente a la mupirocina (SARM-R-MUP) y su capacidad de producir biofilm en los pacientes del Hospital de Pediatría Garrahan.

\section{METODOLOGÍA}

Se llevó a cabo un estudio descriptivo por análisis de muestras de sangre a través de una conveniente estrategia de muestreo de pacientes con bacteriemia en el período de enero de 2011 a diciembre de 2015. El Hospital Garrahan es un hospital pediátrico líder en Argentina y referencial en la región, que recibe a niños de países limítrofes, como Bolivia, Perú y Paraguay, ubicado en la Ciudad de Buenos Aires, Argentina. La aprobación ética para este estudio fue determinada por la Junta de Revisión Institucional y el Comité de Ética del Hospital Garrahan (protocolo n. ${ }^{\circ}$ 868). Se obtuvo una muestra de sangre por paciente y se realizó la identificación de $S$. aureus en el laboratorio del Servicio de Microbiología del Hospital Garrahan de acuerdo con las especificaciones del Instituto de Estándares Clínicos y de Laboratorio. ${ }^{9} \mathrm{La}$ confirmación de SARM se realizó por la identificación de los perfiles proteicos específicos mediante espectrometría de masas desorción/ ionización láser asistida por matriz-tiempo de vuelo (matrix assisted laser desorption ionization time- of-flight; MALDI/TOF, por sus siglas en inglés) (bioMérieux, Francia). La susceptibilidad a los antibióticos de los aislados se realizó mediante el método de difusión en disco para los siguientes antibióticos: oxacilina, cefoxitina, eritromicina, clindamicina, teicoplanina, gentamicina, ciprofloxacina, levofloxacina, quinupristina/ dalfopristina, linezolid, minociclina, rifampicina y trimetoprima/sulfametoxazol, según las recomendaciones y la clasificación del European Committee on Antimicrobial Susceptibility Testing (EUCAST). Las pruebas de sensibilidad fueron confirmadas por VITEK2 (bioMérieux). La susceptibilidad a la mupirocina (Forberton Co. Laboratories) se realizó mediante el método de difusión en discos Kirby-Bauer, y su nivel de resistencia, por determinación de la CIM. Se ensayaron concentraciones finales entre 0,062 y $2,048 \mu \mathrm{g} / \mathrm{ml}$ a través del método de microdilución en caldo. ${ }^{10}$

La detección del biofilm bacteriano se llevó a cabo por la técnica de tinción con cristal violeta (CV) en placas de cultivo de 96 pocillos mediante la determinación de la densidad óptica (DO). La producción de biofilm se calculó considerando una DO crítica (DOc) igual a 0,1 , que correspondió a la media aritmética de la absorbancia de los controles negativos más tres veces el desvío estándar. ${ }^{11}$ La clasificaron fue la siguiente: aislados no productores de biofilm ( $\mathrm{DO} \leq \mathrm{DOc})$; productores débiles de biofilm ( $\mathrm{DOc}<\mathrm{DO} \leq 2$ DOc); productores moderados de biofilm (2 DOc

TABLA 1. Perfiles de resistencia cruzada de los antibióticos y concentraciones inhibitorias minimas de la mupirocina de aislados clínicos de Staphylococcus aureus resistente a la meticilina

\begin{tabular}{|c|c|c|c|c|c|c|c|c|c|c|c|}
\hline & \multicolumn{2}{|c|}{ Mupirocina } & \multirow[t]{2}{*}{ Oxaciclina } & \multirow[t]{2}{*}{ Cefoxitina } & \multirow{2}{*}{$\frac{\text { Eritromicina }}{\mathrm{KB}^{*}}$} & \multirow[t]{2}{*}{ Clindamicina } & \multirow[t]{2}{*}{ Gentamicina } & \multirow[t]{2}{*}{ Ciprofloxacina } & \multirow[t]{2}{*}{ Levofloxacina } & \multirow[t]{2}{*}{ Linezolid } & \multirow[t]{2}{*}{ Rifampicina } \\
\hline & $\mathrm{KB}^{*}$ & $\mathrm{CIM}^{* *}$ & & & & & & & & & \\
\hline GM-36 & $\mathrm{R}$ & $>1024$ & $\mathrm{R}$ & $\mathrm{R}$ & $\mathrm{R}$ & $\mathrm{R}$ & S & $\mathrm{R}$ & S & $S$ & S \\
\hline GM-42 & $\mathrm{R}$ & $\geq 512$ & $\mathrm{R}$ & $\mathrm{R}$ & $\mathrm{R}$ & $\mathrm{R}$ & $\mathrm{R}$ & S & $S$ & S & S \\
\hline GM-43 & $\mathrm{R}$ & $\geq 512$ & $\mathrm{R}$ & $\mathrm{R}$ & $\mathrm{R}$ & $\mathrm{R}$ & $\mathrm{R}$ & $S$ & $S$ & S & S \\
\hline GM-134 & $\mathrm{R}$ & $\geq 512$ & $\mathrm{R}$ & $\mathrm{R}$ & $\mathrm{R}$ & $\mathrm{R}$ & $\mathrm{R}$ & $S$ & $S$ & S & S \\
\hline GM-212 & $\mathrm{R}$ & $\geq 512$ & $\mathrm{R}$ & $\mathrm{R}$ & $\mathrm{R}$ & $\mathrm{R}$ & $\mathrm{R}$ & S & S & S & S \\
\hline GM-4 & S & 0,125 & $\mathrm{R}$ & $\mathrm{R}$ & $\mathrm{R}$ & $\mathrm{R}$ & $\mathrm{R}$ & $\mathrm{R}$ & I & $S$ & $S$ \\
\hline GM-17 & $S$ & 0,125 & $\mathrm{R}$ & $\mathrm{R}$ & $\mathrm{R}$ & $\mathrm{R}$ & $\mathrm{R}$ & $\mathrm{R}$ & I & S & I \\
\hline GM-20 & $S$ & 0,125 & $\mathrm{R}$ & $\mathrm{R}$ & $\mathrm{R}$ & $\mathrm{R}$ & $S$ & $S$ & $S$ & S & $S$ \\
\hline GM-31 & S & 0,125 & $\mathrm{R}$ & $\mathrm{R}$ & $\mathrm{R}$ & $\mathrm{R}$ & $\mathrm{R}$ & $\mathrm{R}$ & I & S & I \\
\hline GM-34 & $S$ & 0,250 & $\mathrm{R}$ & $\mathrm{R}$ & $\mathrm{R}$ & $\mathrm{R}$ & $\mathrm{R}$ & $\mathrm{R}$ & $\mathrm{R}$ & $\mathrm{R}$ & I \\
\hline
\end{tabular}

* Kirby-Bauer; ${ }^{* *}$ CIM fue medida por la técnica de microdilución $(\mu \mathrm{g} / \mathrm{ml})$; SARM: S. aureus resistente a la meticilina; GM: muestra del Hospital Garrahan; R: resistencia; S: susceptibilidad; I: resistencia intermedia. 
$<\mathrm{DO} \leq 4 \mathrm{DOc})$ y fuertes productores de biofilm (4 DOc $<$ DO). Los experimentos se informaron como media \pm desvió estándar, y la significación estadística (valor de $\mathrm{p}<0,05$ ) se realizó utilizando el software InfoStat 2015.

\section{RESULTADOS}

Se analizaron 217 aislados clínicos de SARM, en los cuales se detectó un 2,3\% (5/217) de aislados resistentes a la mupirocina mediante la técnica de Kirby-Bauer (Tabla 1). Además de la resistencia a la oxaciclina y a la cefoxitina, los aislados clínicos mostraron resistencias moderadas a la eritromicina, a la clindamicina y a la gentamicina $(27,6 \%, 25 \%$, $15,2 \%$, respectivamente) y un bajo nivel de resistencia a la ciprofloxacina, a la levofloxacina, a la trimetoprima/sulfametoxazol y al linezolid $(6,9 \%, 1,4 \%, 0,5 \%$ y $0,5 \%$, respectivamente).

En la Tabla 1, se resumen los perfiles de resistencia cruzada de los aislados clínicos de SARM-R-MUP denominados SARM GM-36, 42, 43, 134 y 212. Además, se incluyen los valores de CIM de la mupirocina determinados por el método de microdilución en caldo. Todos los aislados clínicos de SARM-R-MUP tuvieron un valor de CIM > $512 \mu \mathrm{g} / \mathrm{ml}$ y mostraron resistencia cruzada con la clindamicina, la eritromicina, la gentamicina y la ciprofloxacina. En comparación, se muestran aislados susceptibles a la mupirocina medidos bajo las mismas condiciones experimentales, los cuales mostraron valores de CIM esperables para bacterias susceptibles al antibiótico $(0,12-0,25 \mu \mathrm{g} / \mathrm{ml})$.

FIGURA 1. Detección de la producción de biofilm en los aislados clínicos de Staphylococcus aureus resistente a la meticilina y resistentes o susceptibles a la mupirocina mediante la tinción por cristal violeta a una absorbancia de $595 \mathrm{~nm}$

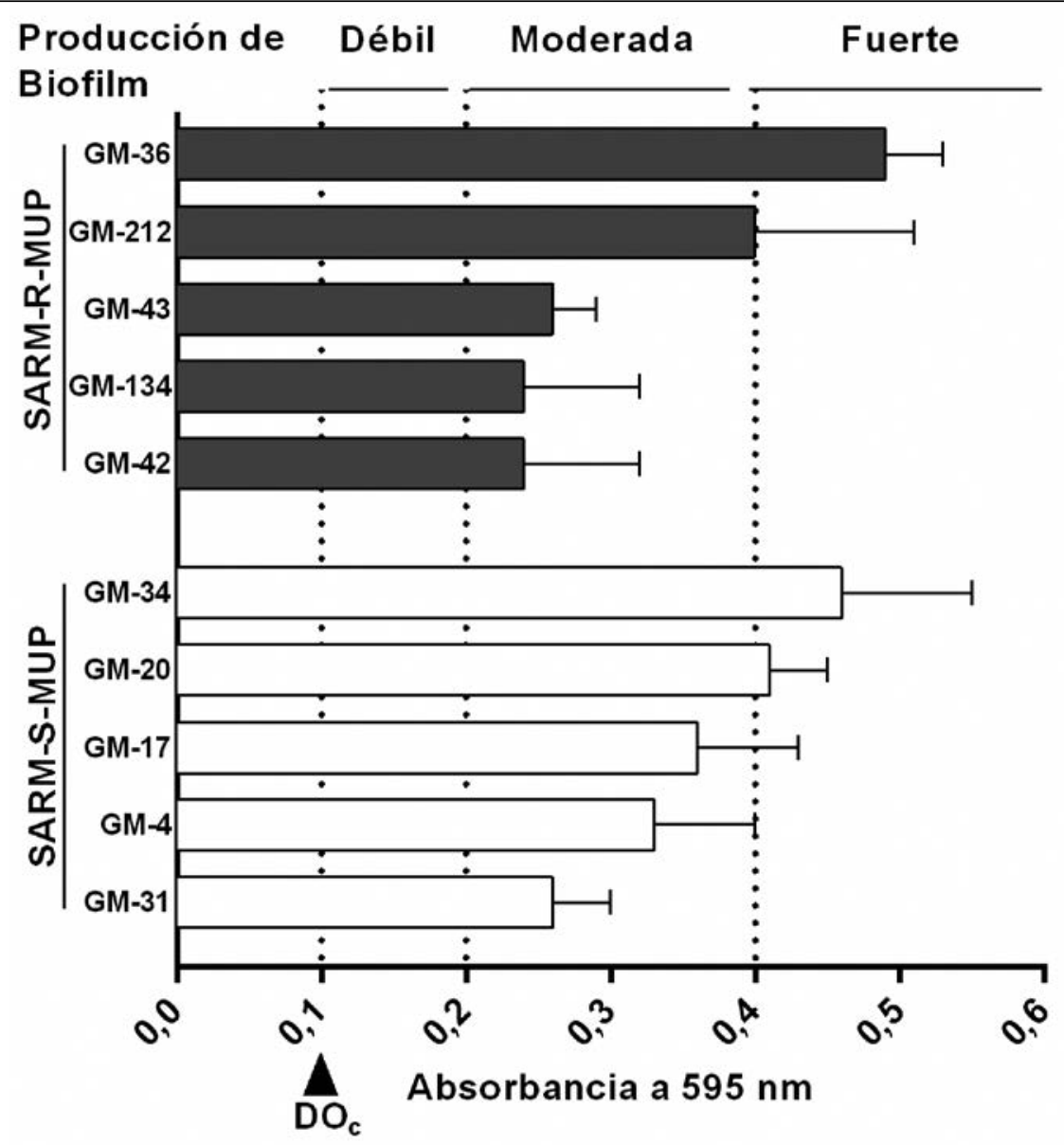

La producción del biofilm fue débil, moderada o fuerte considerando una densidad óptica crítica (DOc) = 0,1, ilustrada por líneas de puntos verticales. S. aureus resistente a la meticilina (SARM) y resistentes o susceptibles a la mupirocina: SARM-RMUP o SARM-S-MUP, respectivamente; DOc: densidad óptica crítica; GM: muestra del Hospital Garrahan. 
Además, se estudió si los SARM-R-MUP tenían la capacidad de producir biofilm. Los resultados mostraron que todos los aislados de SARM-R-MUP (GM-36, 42, 43, 134 y 212) fueron productores del biofilm (Figura 1). Entre ellos, tres aislados formaron una moderada cantidad de biofilm, mientras que los otros dos fueron fuertes productores. Cuando se analizaron cinco aislados susceptibles a la mupirocina (SARM-SMUP) que presentaban entre 4 y 9 resistencias a antibióticos (GM-4, 17, 20, 31 y 34), se observó que fueron también capaces de producir el biofilm en un nivel similar a los aislados resistentes a la mupirocina.

\section{DISCUSIÓN}

En este trabajo se reporta, por primera vez en Argentina, la emergencia de la resistencia a la mupirocina en el SARM, en los pacientes pediátricos. Si bien la incidencia es baja $(2,7 \%)$, es importante destacar el alto nivel de resistencia cruzada de la mupirocina con la eritromicina, la clindamicina y la gentamicina. Para complicar aún más la situación, los aislados de SARMR-MUP fueron capaces de sintetizar biofilm y presentaron de moderados a altos niveles de producción de este.

Otros autores reportaron un alto nivel de resistencias simultáneas de la mupirocina con otros antibióticos asociado con una recolonización más habitual por SARM. ${ }^{4-12}$ Por ello, la mupirocina se recomienda como tratamiento de primera línea para el impétigo en los pacientes con un número limitado de lesiones y, sobre todo, teniendo en cuenta los patrones locales de resistencia del SARM según las directrices de 2014 de la Sociedad de Enfermedades Infecciosas. ${ }^{3}$ En un hospital de niños de EE. UU., se detectó la resistencia a la mupirocina en el 14,7\% de los aislamientos de $S$. aureus asociados con infecciones recurrentes de la piel y los tejidos blandos, tasa que fue más alta que la determinada en otros estudios comunitarios de niños $(1,8 \%) .{ }^{4}$ En los pacientes ambulatorios con afecciones dermatológicas en EE. UU., se detectó la resistencia a la mupirocina en S. aureus asociada con dermatitis atópica, epidermólisis bullosa e inmunosupresión como factores de riesgo adicionales, con una incidencia del 19,3\% al 31,3\%. ${ }^{5}$

Es bien conocido que, en los pacientes con sepsis, el uso de los dispositivos médicos, como los catéteres venosos centrales, es común y puede favorecer el crecimiento del biofilm bacteriano. Nuestros resultados mostraron que todos los aislados de SARM-R-MUP eran productores de biofilm. Con respecto a la posible correlación entre la resistencia a la mupirocina en los aislados de SARM y su capacidad para formar el biofilm, otros autores reportaron cepas de SARM-RMUP aisladas a través de hisopados de heridas infectadas tomados posoperación, que eran productoras de biofilm. ${ }^{12}$ Se ha sugerido que la formación de biofilm puede jugar un papel en la resistencia a la mupirocina entre las poblaciones de pacientes hospitalizados en general. ${ }^{11,12}$

\section{CONCLUSIÓN}

La presencia de aislados clínicos de SARMR-MUP con capacidad para producir el biofilm en los pacientes hospitalizados dificulta los protocolos de descolonización y puede ser responsable de infecciones crónicas o persistentes, puesto que estos son capaces de sobrevivir en ambientes hostiles dentro del huésped. Por lo tanto, la emergencia de la resistencia a la mupirocina en los aislados de SARM en Argentina es un problema creciente que exige un monitoreo a futuro para seguir su evolución en el tiempo.

\section{Agradecimientos}

Los autores desean agradecer a la Dra. Claudia Hernández, Jefa del Servicio de Microbiología del Hospital Pediátrico Garrahan, por proporcionar las cepas clínicas de SARM.

\section{REFERENCIAS}

1. Paganini HR, Della Latta P, Soto A, et al. Bacteriemias por Staphylococcus aureus adquiridas en la comunidad: 17 años de experiencia en niños de la Argentina. Arch Argent Pediatr. 2010;108(4):311-7.

2. Khokhlova OE, Hung WC, Wan TW, et al. Healthcare- and community-associated methicillin-resistant Staphylococcus aureus (MRSA) and fatal pneumonia with pediatric deaths in Krasnoyarsk, Siberian Russia: Unique MRSA's multiple virulence factors, genome, and stepwise evolution. PLoS One. 2015;10(6):e0128017.

3. Stevens DL, Bisno AL, Chambers HF, et al. Practice guidelines for the diagnosis and management of skin and soft tissueinfections: 2014 Update by the Infectious diseases society of America. Clin Infect Dis. 2014;59(2):147-59.

4. Hogue JS, Buttke P, Braun LE, Fairchok MP. Mupirocin resistance related to increasing mupirocin use in clinical isolates of methicillin-resistant Staphylococcus aureus in a pediatric population. J Clin Microbiol. 2010;48(7):2599-600.

5. Antonov NK, Garzon MC, Morel KD, et al. High prevalence of mupirocin resistance in Staphylococcus aureus isolates from a pediatric population. Antimicrob Agents Chemother. 2015;59(6):3350-6.

6. McCarthy H, Rudkin JK, Black NS, et al. Methicillin resistance and the biofilm phenotype in Staphylococcus aureus. Front Cell Infect Microbiol. 2015;5:1.

7. Gardella N, Murzicato S, Di Gregorio S, et al. Prevalence and characterization of methicillin-resistant Staphylococcus aureus among healthy children in a city of Argentina. Infect Genet Evol. 2011;11(5):1066-71. 
8. Ramos RL, Teixeira LA, Ormonde LR, et al. Emergence of mupirocin resistance in multiresistant Staphylococcus aureus clinical isolates belonging to Brazilian epidemic clone III: B:A. J Med Microbiol. 1999;48(3):303-7.

9. Clinical and Laboratory Standards Institute. Performance for antimicrobial susceptibility testing. $26^{\text {th }}$ ed. Wayne, PA: CLSI; 2016.

10. Ojeda-Sana AM, Repetto V, Moreno S. Carnosic acid is an efflux pumps modulator by dissipation of the membrane potential in Enterococcus faecalis and Staphylococcus aureus. World J Microbiol Biotechnol. 2013;29(1):137-44.

11. Xu Z, Liang Y, Lin S, et al. Crystal Violet and XTT Assays on Staphylococcus aureus Biofilm Quantification. Curr Microbiol 2016;73(4):474-82.

12. Günther F, Blessing B, Tacconelli E, Mutters N. MRSA decolonization failure-are biofilms the missing link? Antimicrob Resist Infect Control. 2017;6:32.

\title{
Anticuerpos antitransglutaminasa tisular IgA y contra péptidos de gliadina desaminados IgG como predictores de enfermedad celíaca
}

\author{
$\operatorname{Ig} A$ anti-tissue transglutaminase antibodies and IgG antibodies against \\ deamidated gliadin peptides as predictors of celiac disease
}

\author{
Dr. Gonzalo Ortiz ${ }^{a}$ Dra. Gabriela Messere ${ }^{a}$, Dra. María del C. Toca ${ }^{a}$, Dra. Mirian Fioruccib, \\ Dr. Román Bigliardi ${ }^{a}$, Dr. Jorge Vidal ${ }^{a}$ y Dr. Ricardo Reynoso ${ }^{a}$
}

\section{RESUMEN}

Objetivo. Comparar el rendimiento de anticuerpos antitransglutaminasa $\operatorname{Ig} \mathrm{A}$ (anti-TG2 $\operatorname{Ig} \mathrm{A}$ ), antiendomisio $\operatorname{Ig} \mathrm{A}$ (EMA IgA) y antigliadina desaminada IgA/IgG (AGADGP IgA/IgG) para el diagnóstico de enfermedad celiaca.

Métodos. Estudio descriptivo en pacientes con enfermedad celíaca. Se dosaron anticuerpos: AGADGP (IgA/IgG), EMA IgA, anti-TG2 IgA y biopsia intestinal. Sexo: mujeres (61\%). Mediana de edad: 78,4 meses.

Resultados. Se incluyeron 136 niños; 108 presentaron AGADGP IgA elevado; 124, AGADGP IgG aumentado; 128, EMA IgA positivo; 130, anti-TG2 IgA aumentado. Cuatro de 6 pacientes con anti-TG2 IgA negativos tenían AGADGP IgG elevado.

La combinación de los anticuerpos AGADGP IgG + antiTG2 IgA tuvo una correlación positiva en 134 pacientes y la combinación AGADGP IgG + EMA fue positiva en 133 niños. Conclusión. Se demostró la buena especificidad y sensibilidad de EMA IgA, anti-TG2 IgA y AGADGP IgG. La combinación AGADGP IgG/anti-TG2 mostró sensibilidad del 98-99 \% y especificidad del $100 \%$. La elección de anti-TG2 y AGADGP IgG da excelentes resultados, con bajo costo y no depende del operador.

Palabras clave:enfermedad celiaca, anticuerpos, diagnóstico, niños.

a. Sección de Gastroenterología, Hepatología y Nutrición Infantil, Servicio de Pediatría.

b. Servicio de Laboratorio.

Hospital Nacional Alejandro Posadas.

Correspondencia:

Dr. Gonzalo Ortiz: ortizgonzalojavier@gmail.com

Financiamiento: Ninguno.

Conflicto de intereses: Ninguno que declarar.

Recibido: 6-9-2017.

Aceptado: 15-8-2018 http:/ / dx.doi.org/10.5546/ aap.2019.52

Texto completo en inglés:

http:/ / dx.doi.org/10.5546/ aap.2019.eng.52

Cómo citar: Ortiz G, Messere G, Toca MC, Fiorucci M, et al. Anticuerpos antitransglutaminasa tisular IgA y contra péptidos de gliadina desaminados IgG como predictores de enfermedad celiaca. Arch Argent Pediatr 2019;117(1):52-55.

\section{INTRODUCCIÓN}

La enfermedad celíaca (EC) es una enfermedad crónica inmunomediada, sistémica, precipitada por la ingestión de granos que contienen gluten, que afecta al intestino delgado de individuos genéticamente predispuestos. ${ }^{1}$

Los anticuerpos contra proteínas propias (autoanticuerpos) son marcadores de autoinmunidad; pueden ser anti-transglutaminasa (anti-TG2) o antiendomisio (EMA). ${ }^{2-4}$

El anticuerpo EMA es el más específico. Este test presenta una alta sensibilidad (90-98 \%) y especificidad, realizado por inmunofluorescencia indirecta (IFI) y observador dependiente, por lo cual se utiliza en el algoritmo diagnóstico como test confirmatorio.

El anti-TG2 se realiza por el método de ensayo por inmunoabsorción ligado a enzimas (enzymelinked immunosorbent assay; ELISA, por sus siglas en inglés), que es más objetivo y sencillo, y se recomienda como herramienta inicial en el algoritmo diagnóstico de la EC. ${ }^{2-4}$

Se ha demostrado que la sensibilidad del 